\title{
Contributions to the Study of Dynamic Absorbers, a Case Study
}

\author{
Monica Bălcău ${ }^{1}$, Angela Pleşa ${ }^{1}$, Dan Opruţa ${ }^{1}$ \\ ${ }^{1}$ Technical University of Cluj-Napoca, Automotive Engineering Department, Cluj-Napoca, B-dul Muncii 103-105 \\ Correspondence to: Monica.Balcau@auto.utcluj.ro
}

\begin{abstract}
Dynamic absorbers are used to reduce torsional vibrations. This paper studies the effect of a dynamic absorber attached to a mechanical system formed of three reduced masses which are acted on by one, two or three order $\mathrm{x}$ harmonics of a disruptive force.
\end{abstract}

Keywords: dynamic absorber, torsional vibrations, reduced masses.

\section{Introduction}

This paper studies the effect of dynamic absorbers on reducing torsional vibrations.

Like the inertial effects, the forces and the torque produced by thermal processes in the cylinders of internal combustion engines produce forces and torsional moments that vary nonlinearly.

These forces, applied to the crankshaft and to the engine block, produce translational and rotational oscillations of the engine block and torsional oscillations in the crankshaft. They have to be reduced, because they cause noise and vibrations in an engine.

In order to calculate the torsional vibrations of a complex elastic system of this kind, the system must first be turned into a simpler equivalent dynamic system, formed of an elastic linear shaft of negligible mass loaded with circular reduced masses obtained by reducing the mobile gears.

This study focuses on three mechanical systems formed of three reduced masses, which receive a pendulum dynamic absorber. These masses are acted on by one, two or three order $\times$ harmonics, resulted from the decomposition of the Fourier series of the disruptive force with periodic variation. The dynamic absorber is attached at the end of the mechanical system.

\section{A mechanical system acted on by one harmonic}

The first case deals with a mechanical system formed of three reduced masses with a dynamic absorber attached according to Figure 1. The dynamic absorber is placed at the end of the mechanical system. The reduced mass $m_{3}$ is acted on by an order $\mathrm{x}$ harmonic of a disruptive force presenting a periodic variation marked with $P_{x} \cdot \cos \left(\Omega_{x} t-\varepsilon\right)$.
The dynamic absorber is replaced by an equivalent reduced mechanical system formed of the reduced mass $m_{4}$ and the segment of the reduced crankshaft with an elastic constant $c_{34}$.

The elastic constants of the segments of crankshafts between the two consecutive reduced masses and the mechanical axial moments of the reduced masses in relation to the symmetrical geometry axis of the shaft must be chosen in such a way that the kinetic energy and the potential energy of the real vibrating system (formed by the crankshaft and its mobile gears) is equal to the kinetic energy and the potential energy of the reduced vibrating system.

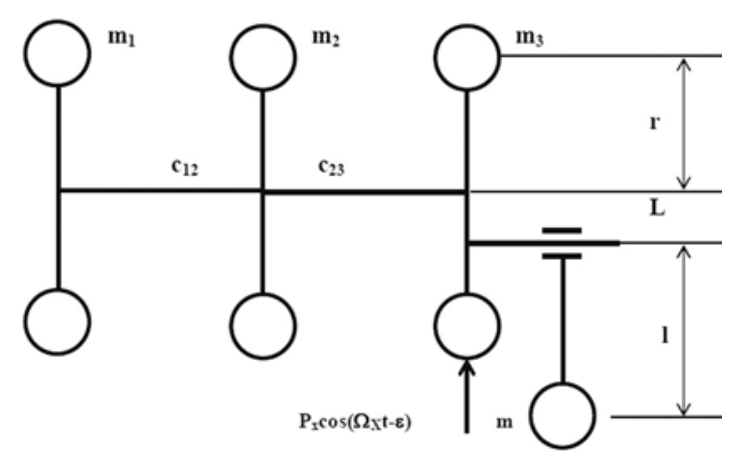

Figure 1: Mechanical system

This case starts from the differential equation system governing the torsional vibrations created by the mechanical system presented in Figure 2:

$$
\begin{aligned}
m_{1} \frac{\mathrm{d}^{2} a_{1}}{\mathrm{~d} t^{2}}+c_{12}\left(a_{1}-a_{2}\right) & =0 \\
m_{2} \frac{\mathrm{d}^{2} a_{2}}{\mathrm{~d} t^{2}}+c_{12}\left(a_{2}-a_{1}\right)+ & \\
c_{23}\left(a_{2}-a_{3}\right) & =0
\end{aligned}
$$




$$
\begin{aligned}
m_{3} \frac{\mathrm{d}^{2} a_{3}}{\mathrm{~d} t^{2}}+c_{23}\left(a_{3}-a_{2}\right)+ & \\
c_{34}\left(a_{3}-a_{4}\right) & =P_{x} \cos \left(\Omega_{x} t-\varepsilon\right) \\
m_{4} \frac{\mathrm{d}^{2} a_{4}}{\mathrm{~d} t^{2}}+c_{34}\left(a_{4}-a_{3}\right) & =0
\end{aligned}
$$

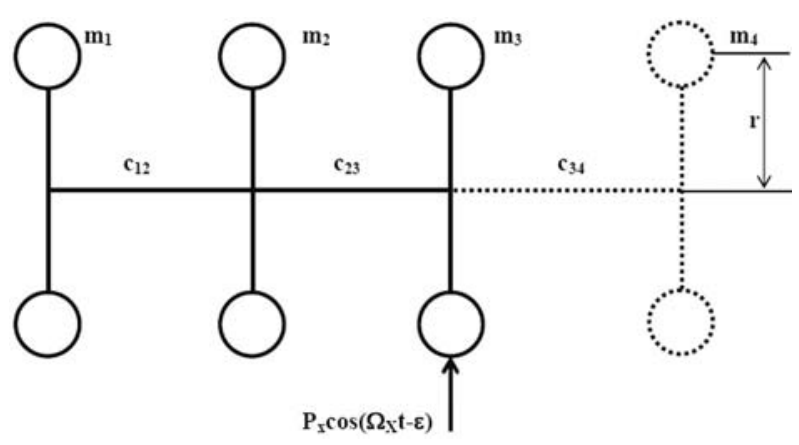

Figure 2: Equivalent mechanical system

The relative linear elongations (displacement) $a_{i}$ measured on the reduced circle of radius $\mathrm{r}$ (of the vibrations of the four reduced masses) are expressed in relation to the linear amplitudes $A_{i}$ :

$$
a_{i}=A_{i} \cos \left(\Omega_{x} t-\varepsilon\right) \quad(i=1 \sim 4)
$$

In order for the mechanical system formed of the reduced mass $m_{4}$ and the shaft segment with elastic constant $c_{34}$ to be dynamically equivalent to the dynamic absorber attached to the reduced mass $m_{3}$ (in other words for this mass $m_{3}$ to be subjected to the same torque as the dynamic absorber), it is necessary and sufficient that:

$$
m_{4}=m \frac{(L+l)^{2}}{r^{2}} \quad c_{34}=m \frac{(L+l)^{2}}{r^{2}} \frac{L}{l} \omega_{0}^{2}
$$

The square value of the order $x$ harmonic is expressed by:

$$
\Omega_{x}^{2}=x^{2} \omega_{0}^{2}
$$

where $x$ represents the order of the harmonic and $\omega_{0}$ the angular speed of the shaft.

Taking into consideration equations (2), (3), (4) and introducing the differential equation system (1), we obtain an algebraic system of equations. By solving this system of equations we get the four determinants. If the dynamic absorber is built in such a way that:

$$
\frac{L}{l}=x^{2}
$$

the expressions of the four determinants become:

$$
\begin{aligned}
& \Delta_{1}=-\frac{P_{x}}{m_{3}} \frac{c_{12}}{m_{1}} \frac{c_{23}}{m_{2}} \omega_{0}^{2}\left(x^{2}-\frac{L}{l}\right) \\
& \Delta_{2}=\frac{P_{x}}{m_{3}} \frac{c_{23}}{m_{2}} \omega_{0}^{2}\left(x^{2} \omega_{0}^{2}-\frac{c_{12}}{m_{1}}\right)\left(x^{2}-\frac{L}{l}\right)
\end{aligned}
$$

$$
\begin{aligned}
\Delta_{3}= & -\frac{P_{x}}{m_{3}} \omega_{0}^{2}\left(x^{2} \omega_{0}^{2}-\frac{c_{12}}{m_{1}}\right) . \\
& \left(x^{2} \omega_{0}^{2}-\frac{c_{23}}{m_{2}}-\frac{c_{12}}{m_{2}}\right)\left(x^{2}-\frac{L}{l}\right) \\
\Delta_{4}= & \frac{P_{x}}{m_{3}} \omega_{0}^{2}\left(x^{2} \omega_{0}^{2}-\frac{c_{12}}{m_{1}}\right) . \\
& \left(x^{2} \omega_{0}^{2}-\frac{c_{23}}{m_{2}}-\frac{c_{12}}{m_{2}}\right) \frac{L}{l} \omega_{0}^{2}
\end{aligned}
$$

An analysis of the four determinants shows that the only mass that executes torsional vibrations is mass $m_{4}$, which is not part of the reduced crankshaft. The other reduced masses do not execute torsional vibrations. So:

$$
A_{1}=0 \quad A_{2}=0 \quad A_{3}=0 \quad A_{4} \neq 0
$$

\section{A mechanical system acted on by two order $\times$ harmonics}

This case deals with a mechanical system composed of three reduced masses, which receives a dynamic absorber placed at the end of the system presented in Figure 3. The reduced masses $m_{2}, m_{3}$ are acted on by two order $\times$ harmonics of the disruptive force presenting a periodic vibration marked with $P_{x} \cos \left(\Omega_{x} t-\varepsilon\right)$.



Figure 3: Mechanical system

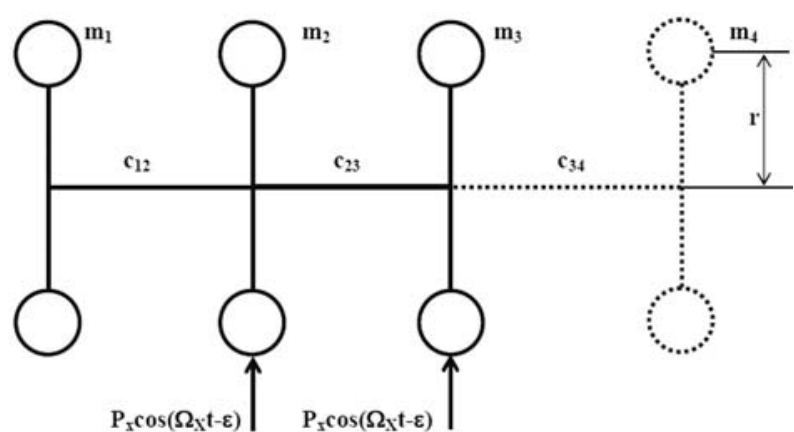

Figure 4: Equivalent mechanical system 
The reduced mass $m_{4}$, together with the shaft segment with elastic constant $c_{34}$, forms a dynamic mechanical system equivalent to the dynamic absorber which applies the same torque to mass $m_{3}$ as the absorber (Figure 4). As in the previous case, the dynamic absorber is replaced by an equivalent dynamic system formed of the reduced mass $m_{4}$ and the reduced crankshaft segment having elastic constant $c_{34}$ (Figure 4).

The study starts from the differential equation system that governs the torsion vibrations performed by the mechanical system represented in Figure 4 :

$$
\begin{aligned}
m_{1} \frac{\mathrm{d}^{2} a_{1}}{\mathrm{~d} t^{2}}+c_{12}\left(a_{1}-a_{2}\right) & =0 \\
m_{2} \frac{\mathrm{d}^{2} a_{2}}{\mathrm{~d} t^{2}}+c_{12}\left(a_{2}-a_{1}\right)+ & \\
c_{23}\left(a_{2}-a_{3}\right) & =P_{x} \cos \left(\Omega_{x} t-\varepsilon\right) \\
m_{3} \frac{\mathrm{d}^{2} a_{3}}{\mathrm{~d} t^{2}}+c_{23}\left(a_{3}-a_{2}\right)+ & \\
c_{34}\left(a_{3}-a_{4}\right) & =P_{x} \cos \left(\Omega_{x} t-\varepsilon\right) \\
m_{4} \frac{\mathrm{d}^{2} a_{4}}{\mathrm{~d} t^{2}}+c_{34}\left(a_{4}-a_{3}\right) & =0
\end{aligned}
$$

Relation (2) gives the elongation expressions $a_{i}$ related to the amplitudes recorded on radius $r$ on the reduction circles. Taking into account expressions (2), (3), (4) and introducing the differential system of equations (8) we obtain a system of algebraic equations. By solving this system, we obtain expressions for the five determinants.

$$
\begin{aligned}
\Delta= & \frac{1}{m_{3}} m \frac{(L+l)^{2}}{r^{2}}\left(\frac{L}{l} \omega_{0}^{2}\right)^{2}\left[\left(x^{2} \omega_{0}^{2}-\frac{c_{12}}{m_{1}}\right) .\right. \\
& \left.\left(x^{2} \omega_{0}^{2}-\frac{c_{12}}{m_{2}}-\frac{c_{23}}{m_{2}}\right)-\frac{c_{12}}{m_{1}} \frac{c_{12}}{m_{2}}\right] \\
\Delta_{1}= & -\frac{P_{x}}{m_{2}} \omega_{0}^{4} \frac{c_{12}}{m_{1}} \frac{1}{m_{3}} m \frac{(L+l)^{2}}{r^{2}}\left(\frac{L}{l}\right)^{2} \\
\Delta_{2}= & -\frac{P_{x}}{m_{2}} \omega_{0}^{4}\left(x^{2} \omega_{0}^{2}-\frac{c_{12}}{m_{1}}\right) \frac{1}{m_{3}} m \frac{(L+l)^{2}}{r^{2}}\left(\frac{L}{l}\right)^{2} \\
\Delta_{3}= & 0 \\
\Delta_{4}= & -\frac{P_{x}}{m_{2}} \frac{c_{23}}{m_{3}} \frac{L}{l} \omega_{0}^{2}\left(x^{2} \omega_{0}^{2}-\frac{c_{12}}{m_{1}}\right)+ \\
& \frac{P_{x}}{m_{3}}\left[\left(x^{2} \omega_{0}^{2}-\frac{c_{12}}{m_{1}}\right)\left(x^{2} \omega_{0}^{2}-\frac{c_{12}}{m_{2}}-\frac{c_{23}}{m_{2}}\right)-\right. \\
& \left.\frac{c_{12}}{m_{1}} \frac{c_{12}}{m_{2}}\right] \frac{L}{l} \omega_{0}^{2}
\end{aligned}
$$

The analysis of the expressions (9) shows that the reduced masses $m_{1}, m_{2}$, and $m_{4}$ - born from a reduction operation - perform torsion vibrations. The only mass that does not perform any torsional vibrations is mass $m_{3}$, which is the mass that has the dynamic absorber attached.

$$
A_{1} \neq 0 \quad A_{2} \neq 0 \quad A_{3}=0 \quad A_{4} \neq 0
$$

\section{The mechanical system acted on by three order $x$ harmonics}

This case investigates a mechanical system composed of three reduced masses which receive a dynamic absorber placed at the end of the system (Figure 5). The reduced masses $m_{1}, m_{2}, m_{3}$ are acted on by means of three order $\mathrm{x}$ harmonics of disruptive forces presenting a periodic variation marked with $P_{x} \cos \left(\Omega_{x} t-\varepsilon\right)$.



Figure 5: Mechanical system

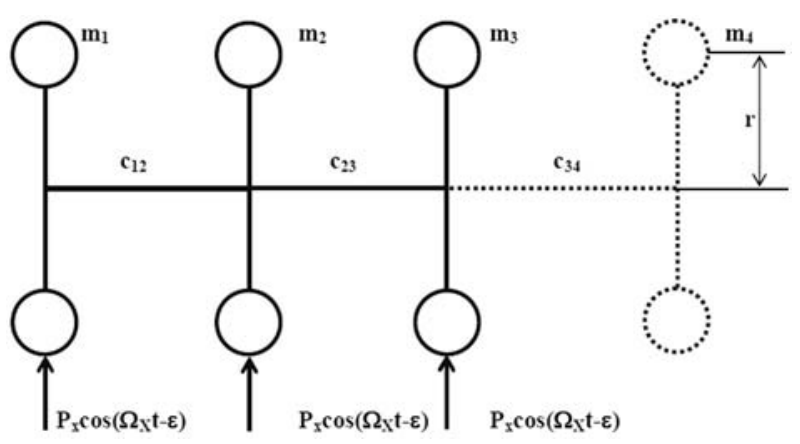

Figure 6: Equivalent mechanical system

Just as in the previous paragraph, the dynamic absorber is replaced by an equivalent dynamic system formed of the reduced mass $m_{4}$ and the reduced shaft segment having the elastic constant $c_{34}$ (Figure 6 ). The differential equations governing the vibratory movements of the mechanical system represented in Figure 6 are:

$$
\begin{aligned}
m_{1} \frac{\mathrm{d}^{2} a_{1}}{\mathrm{~d} t^{2}}+c_{12}\left(a_{1}-a_{2}\right) & =P_{x} \cos \left(\Omega_{x} t-\varepsilon\right) \\
m_{2} \frac{\mathrm{d}^{2} a_{2}}{\mathrm{~d} t^{2}}+c_{12}\left(a_{2}-\right. & \\
\left.a_{1}\right)+c_{23}\left(a_{2}-a_{3}\right) & =P_{x} \cos \left(\Omega_{x} t-\varepsilon\right) \\
m_{3} \frac{\mathrm{d}^{2} a_{3}}{\mathrm{~d} t^{2}}+c_{23}\left(a_{3}-a_{2}\right)+ & \\
c_{34}\left(a_{3}-a_{4}\right) & =P_{x} \cos \left(\Omega_{x} t-\varepsilon\right)
\end{aligned}
$$




$$
m_{4} \frac{\mathrm{d}^{2} a_{4}}{\mathrm{~d} t^{2}}+c_{34}\left(a_{4}-a_{3}\right)=0
$$

The expressions of elongations $a_{i}$ according to the amplitudes recorded on the radius $\mathrm{r}$ reduction circles are given by relation (2).

Taking into account expressions (2), (3), (4) and introducing the differential system of equations (11), we obtain an algebraic system of equations. Solving this system provides the four determinants.

$$
\begin{aligned}
\Delta= & -\frac{1}{m_{3}} m \frac{(L+l)^{2}}{r^{2}}\left(\frac{L}{l} \omega_{0}^{2}\right)^{2}\left[\left(x^{2} \omega_{0}^{2}-\frac{c_{12}}{m_{1}}\right) .\right. \\
& \left.\left(x^{2} \omega_{0}^{2}-\frac{c_{12}}{m_{2}}-\frac{c_{23}}{m_{2}}\right)-\frac{c_{12}}{m_{1}} \frac{c_{12}}{m_{2}}\right] \\
\Delta_{1}= & \frac{P_{x}}{m_{1}}\left(x^{2} \omega_{0}^{2}-\frac{c_{12}}{m_{2}}-\frac{c_{23}}{m_{2}}\right) \frac{1}{m_{3}} m \frac{(L+l)^{2}}{r^{2}} . \\
\Delta_{2}= & -\frac{P_{x}}{m_{1}} \frac{c_{12}}{m_{2}} \frac{1}{m_{3}} m \frac{(L+l)^{2}}{r^{2}}\left(\frac{L}{l} \omega_{0}^{2}\right)^{2}+ \\
& \frac{P_{x}}{m_{2}} \frac{1}{m_{3}} m \frac{(L+l)^{2}}{m_{1}}\left(\frac{L}{m_{1}} \omega_{0}^{2}\right)^{2} \\
r_{3}^{2} & \frac{(L+l)^{2}}{r^{2}}\left(\frac{L}{l}\right)^{2} \\
\Delta_{3}= & 0
\end{aligned}
$$

We observe that:

$$
A_{1} \neq 0 \quad A_{2} \neq 0 \quad A_{3}=0
$$

This means that the only mass that does not execute torsional oscillations is mass $m_{3}$, which received a dynamic absorber. Since mass $m_{4}$ is not a result of reducing the crankshaft, it was no longer useful when calculating the amplitude vibration for this mass.

\section{Conclusion}

The three cases discussed here offer us the following information:
- In the case of the mechanical system formed of three reduced masses acted on by a single order $\mathrm{x}$ harmonic of the disruptive force, if the dynamic absorber is built according to relation (5), the three reduced masses $m_{1}, m_{2}$ and $m_{3}$ do not perform any torsional vibrations, irrespective of the value of the angular speed.

- In the case of the mechanical systems formed of three reduced masses acted on by two and three order $\mathrm{x}$ harmonics of the disruptive force, respectively, if the dynamic absorber is built according to relation (5), the only mass which does not execute torsional vibrations is the mass which receives the dynamic absorber.

\section{References}

[1] Haddow, A. G., Shaw, S. W.: Centrifugal pendulum vibration absorbers: an experimental and theoretical investigation. Nonlinear Dynamics, 34, 293-307, 2003. 2004 Kluwer Academic Publishers. Printed in the Netherlands.

[2] Ripianu, A., Crăciun, I.: The dynamic and strength calculus of straight and crank shafts. Transilvania Press Publishing House Cluj, 1999.

[3] Kraemer, O.: Drehschwingungsrechnung Berechnung der Eigenschwingungszahlen, In Technishe Hochschule Karlsruhe Lehrstuhl für Kolbenmaschinen und Getriebelehre, 1960.

[4] Pop, A. F., Gligor, R. M., Bălcău, M.: Analising of Vibrations Measurements upon Hand-Arm System and Results Comparison with Theoretical Model, 3rd European Conference on Mechanism Science, Previous EUCOMES 2010 Conferences, September 14-18, 2010, Cluj-Napoca, Romania Mechanisms and Machine Science, Springer Vol. 5, p. 277-284. ISBN 978-90-481-9688-3. 